\title{
de conductivity as a geometric phase
}

\author{
Balázs Hetényi \\ Department of Physics, Bilkent University, TR-06800 Bilkent, Ankara, Turkey
}

(Received 4 November 2012; published 18 June 2013)

\begin{abstract}
The zero-frequency conductivity $\left(D_{c}\right)$, the criterion to distinguish between conductors and insulators, is expressed in terms of a geometric phase. $D_{c}$ is also expressed using the formalism of the modern theory of polarization. The tenet of Kohn [Phys. Rev. 133, A171 (1964)], namely that insulation is due to localization in the many-body space, is refined as follows. Wave functions, which are eigenfunctions of the total current operator, give rise to a finite $D_{c}$ and are therefore metallic. They are also delocalized. Based on the value of $D_{c}$ it is also possible to distinguish purely metallic states from states in which the metallic and insulating phases coexist. Several examples which corroborate the results are presented, as well as a numerical implementation. The formalism is also applied to the Hall conductance, and the quantization condition for zero Hall conductance is derived to be $\frac{e \Phi_{B}}{N h c}=\frac{Q}{M}$, with $Q$ and $M$ as integers.
\end{abstract}

DOI: 10.1103/PhysRevB.87.235123

PACS number(s): 03.65.Vf, 71.30.+h, 72.10.Bg

\section{INTRODUCTION}

What makes conductors conducting and insulators insulating? In classical physics this question is answered by considering the localization of individual charge carriers. Localized, bound charges do not contribute to conduction. Quantum mechanics has rendered the answering of this question more difficult. In band theory, conduction can be attributed to the density of electron states at the Fermi level: if $\rho\left(\epsilon_{F}\right) \neq 0$, the system is conducting; if $\rho\left(\epsilon_{F}\right)=0$, it is insulating. However, simple band theory is not able to explain insulation of strongly correlated systems. In 1964 Kohn suggested ${ }^{1}$ that the criterion that distinguishes metals from insulators is localization of the total position of all charge carriers. Kohn also derived $^{1}$ the quantum criterion of dc conductivity, the Drude weight $\left(D_{c}\right)$.

For several decades, testing Kohn's hypothesis was difficult, due to the fact that in crystalline systems (systems with periodic boundary conditions) the total position operator is ill defined. This limitation was overcome by the modern theory of polarization, ${ }^{2-5}$ in which the expectation value of the total position is expressed in terms of a geometric phase. ${ }^{6-8}$ The geometric phase arises upon varying the crystal momentum across the Brillouin zone. In numerical applications the polarization is easiest to calculate in terms of the ground state expectation value of the total momentum shift operator. ${ }^{9,10}$ These developments have simplified the calculation of the polarization considerably, and are now in widespread use in electronic structure calculations.

Moulopoulos and Ashcroft ${ }^{11}$ have also suggested a connection between conduction and a Berry phase related to the center of mass. Recently, the author has shown ${ }^{12}$ that the total current can be expressed as a phase associated with moving the total position across the periodic cell, and that it can be written as a ground state expectation value of the total position shift operator. We note that topological invariants can also characterize metals ${ }^{13}$ as well as insulators.

\section{PURPOSE}

We demonstrate that $D_{c}$ can also be expressed in terms of a geometric phase. The formal expression for $D_{c}$ derived here consists of an expectation value of single-body operators and a geometric phase arising from the variation of the total momentum and the total position. Its form is similar to that of the Hall conductance. ${ }^{14}$ The second term is also expressed in terms of the total momentum and total position shift operators, in other words, based on a formalism similar to that of the modern theory of polarization. The resulting formula establishes the precise connection between localization and conductivity as suggested by Kohn. ${ }^{1}$ If the ground state wave function of a system is an eigenstate of the total current operator, $D_{c}$ is finite. Such wave functions are also delocalized according to the criterion defined by Resta. ${ }^{9,10}$ The calculation of the Drude weight is also straightforward: for metals, the $D_{c}=\frac{\pi \alpha}{L}[\mathrm{Eq}$. (6), where $L$ denotes the size of the system); for insulators, it is zero. For wavefunctions corresponding to coexistence between metallic and insulating phases it holds that $0<D_{c}<\frac{\pi \alpha}{L}$. One calculates the spread in total current, and if this spread is zero, then $D_{c}=\frac{\pi \alpha}{L}$. These results are independent of dimensionality. The formalism is also used to derive the Hall conductance, ${ }^{14}$ and a quantization condition for that quantity being zero is derived. The condition coincides with the wellknown experimental results for the fractional quantum Hall effect. $^{15}$

\section{DEFINITIONS}

Let $|\Psi\rangle$ denote the ground state wave function of an $N$ particle system. In coordinate space one can write $\Psi\left(x_{1}+\right.$ $\left.X, \ldots, x_{N}+X\right)$, where $X$ denotes a shift of all coordinates, or equivalently one can write in momentum space $\Psi\left(k_{1}+\right.$ $\left.K, \ldots, k_{N}+K\right)$. A wave function can be labeled by $X$ or $K[|\Psi(X)\rangle,|\Psi(K)\rangle]$. One can define the shift operators in position or momentum space as

$$
\begin{aligned}
e^{-i \Delta K \hat{X}}|\Psi(K)\rangle & =|\Psi(K+\Delta K)\rangle, \\
e^{-i \Delta X \hat{K}}|\Psi(X)\rangle & =|\Psi(X+\Delta X)\rangle,
\end{aligned}
$$

where $\hat{X}=\sum_{i=1}^{N} \hat{x}_{i}$ and $\hat{K}=\sum_{i=1}^{N} \hat{k}_{i}$. In lattice models the current operator in momentum space takes the form $\hat{K}=$ $\sum_{i=1}^{N} \sin \left(\hat{k}_{i}\right)$. The explicit construction of the shift operators is given in Refs. 12 and 16. 


\section{MAIN RESULTS}

\section{A. Conductivity as a geometric phase}

The Drude weight ${ }^{1}$ is defined as

$$
D_{c}=\frac{\pi}{L} \frac{\partial^{2} E(0)}{\partial \Phi^{2}}
$$

where $\Phi$ denotes a perturbing field, and the derivative is the adiabatic derivative. The second derivative with respect to $\Phi$ can be expressed as

$$
\frac{\partial^{2} E(0)}{\partial \Phi^{2}}=\alpha+\gamma
$$

where

$$
\alpha=i \sum_{j}^{N}\left\langle\Psi\left|\left[\hat{\partial}_{k_{j}}, \hat{\partial}_{x_{j}}\right]\right| \Psi\right\rangle,
$$

and where

$$
\gamma=-\frac{i}{2 \pi} \int_{-\pi / L}^{\pi / L} \int_{0}^{L} d K d X\left[\left\langle\partial_{K} \Psi \mid \partial_{X} \Psi\right\rangle-\left\langle\partial_{X} \Psi \mid \partial_{K} \Psi\right\rangle\right] .
$$

This expression is derived in Appendix B. $\gamma$ has the form of an integrated Berry curvature over a surface in the twodimensional space $K-X$, and can be converted into a geometric phase by application of the Stokes theorem. Note that the Drude weight is the sum of two terms, one proportional to the sum of the commutators of each momentum and position, and a "commutator" of the variables related to the total position and total momentum of the system.

\section{B. Analog of $D_{c}$ based on the modern theory of polarization}

$D_{c}$, in particular the term $\gamma$, can also be expressed using total momentum and total position shift operators. For charge carriers with mass one, the one-body term is

$$
\alpha= \begin{cases}N & \text { for continuous models }, \\ -\frac{\langle\Psi|\hat{T}(0)| \Psi\rangle}{2} & \text { for lattice systems. }\end{cases}
$$

The geometric phase term can be written as

$$
\begin{aligned}
\gamma= & -\lim _{\Delta X, \Delta K \rightarrow 0} \frac{1}{\Delta X \Delta K}\left[\operatorname{Im} \ln \frac{\left\langle\Psi\left|e^{i \Delta K \hat{X}} e^{i \Delta X \hat{K}}\right| \Psi\right\rangle}{\left\langle\Psi\left|e^{i \Delta X \hat{K}}\right| \Psi\right\rangle}\right. \\
& \left.+\operatorname{Im} \ln \frac{\left\langle\Psi\left|e^{i \Delta X \hat{K}} e^{-i \Delta K \hat{X}}\right| \Psi\right\rangle}{\left\langle\Psi\left|e^{i \Delta X \hat{K}}\right| \Psi\right\rangle}\right] .
\end{aligned}
$$

This expression is derived in Appendix C.

\section{INTERPRETATION}

The first term of $D_{c}$, proportional to $\alpha$, is an extensive quantity, a sum over single-body operators. For any nontrivial system it is expected to be finite. For an insulator, the manybody term (proportional to $\gamma$ ) must cancel the single-body term.

We consider a general wave function of the form $\Psi\left(x_{1}, \ldots, x_{N}\right)$ corresponding to an unperturbed ground state. Acting on this function with the shift operators according to the first and second terms of $\gamma$ [Eq. (7)], respectively, results in

$$
\begin{aligned}
& e^{i \Delta K \hat{X}} e^{i \Delta X \hat{K}} \Psi\left(x_{1}, \ldots, x_{N}\right) \\
& \quad=e^{i N \Delta K \Delta X} e^{i \Delta K \sum_{i=1}^{N} x_{i}} \Psi\left(x_{1}+\Delta X, \ldots, x_{N}+\Delta X\right), \\
& e^{i \Delta X \hat{K}} e^{-i \Delta K \hat{X}} \Psi\left(x_{1}, \ldots, x_{N}\right) \\
& \quad=e^{-i \Delta K \sum_{i=1}^{N} x_{i}} \Psi\left(x_{1}+\Delta X, \ldots, x_{N}+\Delta X\right) .
\end{aligned}
$$

Evaluating the scalar products, one can then show that apart from the term $e^{i N \Delta K \Delta X}$ in Eq. (8) the two terms in Eq. (7) are complex conjugates of each other. The term $e^{i N \Delta K \Delta X}$ gives a contribution of $-N$ to the conductivity cancelling the single-body term. When this derivation is valid the system is insulating. This derivation, of course, has limits of validity, for example, if discontinuities are present in the momentum distribution. $^{17}$

If the function $|\Psi\rangle$ is an eigenfunction of the current operator, then $\gamma$ is zero; hence the system is metallic. To show this, one considers that the eigenvalue of the current operator for an unperturbed ground state is zero, which means that the total position shift operator will have no effect at all. In this case the two terms of $\gamma$ are complex conjugates of each other, and their sum will have no imaginary part.

If a wave function is an eigenstate of the total current operator, it also follows that the system is delocalized. Indeed the localization criterion defined by $\operatorname{Resta}^{9,10}$ is

$$
\sigma_{X}^{2}=-\frac{2}{\Delta K^{2}} \operatorname{Re} \ln \left\langle\Psi\left|e^{-i \Delta K \hat{X}}\right| \Psi\right\rangle .
$$

The function resulting from the total momentum shift operator acting on an eigenfunction of the total current will be orthogonal to the original function, resulting in a divergent $\sigma_{X}^{2}$.

To decide whether a particular ground state eigenfunction is an eigenfunction of the current one can calculate the spread in current, ${ }^{12}$ defined as

$$
\sigma_{K}^{2}=-\frac{2}{\Delta X^{2}} \operatorname{Re} \ln \left\langle\Psi\left|e^{-i \Delta X \hat{K}}\right| \Psi\right\rangle .
$$

If $\sigma_{K}$ is zero, then the wave function is indeed a current eigenstate and the system is metallic; moreover, $\gamma=0$ and the $D_{c}=\frac{\pi \alpha}{L}$. Otherwise, the wave function corresponds to an insulating state. To show this one can use the fact that for an eigenfunction of the current with eigenvalue zero the expectation value $\left\langle\Psi\left|e^{-i \Delta X \hat{K}}\right| \Psi\right\rangle=1$ must give one, but for any other case $\left\langle\Psi\left|e^{-i \Delta X \hat{K}}\right| \Psi\right\rangle<1$. In calculating conductivity, one can also use Eq. (9), but this quantity is expected to diverge when the system becomes metallic; hence calculations based on $\sigma_{K}$ can be expected to be more stable.

We remark that a wave function could be a linear combination of an eigenstate of the current operator and a localized state corresponding to the coexistence of the insulating and metallic states. In this case, the single-body term will be partially canceled by the many-body term and a finite Drude weight will result.

\section{EXAMPLES}

\section{A. Fermi sea, BCS}

For both the Fermi sea and BCS wave functions $D_{c}=\frac{\pi \alpha}{L}$. The Fermi sea is diagonal in the momentum representation and corresponds to an eigenstate of $\hat{K}$ with eigenvalue zero. A BCS 
wave function consists of a linear combination of wave functions with different number of particles, but all have eigenvalue of $\hat{K}=0$, and the argument for the Fermi sea extends.

\section{B. Gutzwiller metal}

The Gutzwiller variational wave function was proposed to understand the Hubbard model, ${ }^{18-20}$ and is of the form

$$
\left|\Psi_{G}(\tilde{\gamma})\right\rangle=e^{-\tilde{\gamma} \sum_{i} \hat{n}_{i \uparrow} \hat{n}_{i \downarrow}}|F S\rangle .
$$

The state $|F S\rangle$ denotes the Fermi sea, out of which doubly occupied sites are projected out via the projector $e^{-\tilde{\gamma} \sum_{i} \hat{n}_{i \uparrow} \hat{n}_{i \downarrow}}$. This wave function has been shown ${ }^{21,22}$ to be metallic for finite values of the variational parameter $\tilde{\gamma}\left(D_{c}=\frac{\alpha \pi}{L}\right)$.

Indeed, the geometric phase term $\gamma$ vanishes. To see this, consider that the shift operator $e^{i \Delta X \hat{K}}$ commutes with the projector $e^{-\tilde{\gamma} \sum_{i} \hat{n}_{i \uparrow} \hat{n}_{i \downarrow}}$, since shifting the position of every particle will not affect the number of doubly occupied sites. ${ }^{12}$ Thus $e^{i \Delta X \hat{K}}$ will operate on the Fermi sea, which has eigenvalue $\hat{K}|F S\rangle=0$, and then the same reasoning applies as in the case of the Fermi sea.

\section{Baeriswyl insulating wave function for a spinless system}

An insulating variational solution for spinless fermions on a lattice with nearest neighbor interaction ( $t-V$ model) in one dimension is the Baeriswyl wave function, ${ }^{23}$ which in this case has the form

$$
\left|\Psi_{B}(\tilde{\alpha})\right\rangle=\prod_{\text {RBZ }}\left[e^{-\tilde{\alpha} \epsilon_{k}} c_{k}^{\dagger}+e^{\tilde{\alpha} \epsilon_{k}} c_{k+\pi}^{\dagger}\right]|0\rangle,
$$

where the product is over the reduced Brillouin zone. This wave function is easily shown to be insulating; ${ }^{23}$ hence we expect that it gives $D_{c}=0$.

This can be shown readily by considering again the action of the shift operators on $\left|\Psi_{B}(\tilde{\alpha})\right\rangle$. The scalar products in $\gamma$ evaluate to

$$
\begin{aligned}
& \left\langle\Psi_{B}(\tilde{\alpha})\left|e^{i \Delta K \hat{X}} e^{i \Delta X \hat{K}}\right| \Psi_{B}(\tilde{\alpha})\right\rangle \\
& =\prod_{\operatorname{RBZ}}\left[e^{i \Delta X \sin (k+\Delta K)} e^{-\tilde{\alpha}\left(\epsilon_{k}+\epsilon_{k+\Delta K}\right)}\right. \\
& \left.\quad+e^{-i \Delta X \sin (k+\Delta K)} e^{\tilde{\alpha}\left(\epsilon_{k}+\epsilon_{k+\Delta K}\right)}\right], \\
& \left\langle\Psi_{B}(\tilde{\alpha})\left|e^{i \Delta X \hat{K}} e^{-i \Delta K \hat{X}}\right| \Psi_{B}(\tilde{\alpha})\right\rangle \\
& =\prod_{\mathrm{RBZ}}\left[e^{i \Delta X \sin (k)} e^{-\tilde{\alpha}\left(\epsilon_{k}+\epsilon_{k-\Delta K}\right)}+e^{-i \Delta X \sin (k)} e^{\tilde{\alpha}\left(\epsilon_{k}+\epsilon_{k-\Delta K}\right)}\right] .
\end{aligned}
$$

Substituting into the definition of $\gamma$ and taking the limits $\Delta K, \Delta X \rightarrow 0$ lead to $D_{c}=0$ as expected for an insulating state. The above derivation is also valid for the mean-field spin or charge-density wave solutions of strongly correlated lattice models.
TABLE I. Results from diagonalization of Anderson localization model for a system with 1024 lattice sites and 512 particles. $\Delta K=$ $\Delta X=0.001$.

\begin{tabular}{ccccc}
\hline \hline$U$ & $\sigma_{K}$ & $D_{c} \times L / \pi$ & $-\frac{\langle T\rangle}{2}$ & $\sigma_{X}$ \\
\hline 0 & 0 & 327.95 & 327.95 & \\
1 & $5.8(2)$ & $0.01154(4)$ & $297(7)$ & $38(4)$ \\
2 & $9.8(2)$ & $0.0087(1)$ & $233(3)$ & $17.8(9)$ \\
3 & $12.8(2)$ & $0.0066(2)$ & $175(5)$ & $11.7(5)$ \\
4 & $15.1(2)$ & $0.0051(2)$ & $136(5)$ & $8.4(4)$ \\
5 & $16.7(3)$ & $0.0041(2)$ & $110(5)$ & $6.5(3)$ \\
\hline \hline
\end{tabular}

\section{Anderson localized system}

We have evaluated the above formula for a model which exhibits Anderson localization, ${ }^{24}$ with Hamiltonian of the form

$$
H=-t \sum_{i} c_{i}^{\dagger} c_{i+1}+\text { H.c. }+U \sum_{i} \xi_{i} n_{i},
$$

where $\xi_{i}$ is a number drawn from a uniform Gaussian distribution. By diagonalizing the Hamiltonian we have calculated the localization parameter ${ }^{9,10}$ for different system sizes, and have found that the larger system sizes are always more localized for finite $U$ (results not shown). We have also calculated the Drude weight and the quantity $\sigma_{K}$. The results are shown in Table I.

For the metallic state $\sigma_{K}$ gives zero as expected, and the Drude weight is equal to minus one-half the kinetic energy. For all insulating cases the Drude weight is very near zero, in particular if one compares its magnitude to that of the kinetic energy. While one can calculate the Drude weight directly, this may be difficult in some applications, since phases have to be evaluated. However, evaluating the kinetic energy and the spread in current allows the determination of the Drude weight unambiguously.

\section{HALL CONDUCTANCE}

The Hall conductance can also be expressed in terms of a Berry phase ${ }^{14}$, similar in form to the conductivity derived above (Eq. (5)). It is possible to express the Hall conductance as a ground state observable. ${ }^{25,26}$ Here we express it via shift operators, and derive a quantization condition for zero Hall conductance in a quantum Hall system. The momentum shift operators in this case take forms which are different from those used in expressing dc conductivity.

Our starting point is the form derived by Thouless et al. ${ }^{14}$,

$$
\sigma_{x y}^{H}=\frac{i e^{2}}{2 \pi h} \int d K_{x} d K_{y}\left[\left\langle\partial_{K_{x}} \Psi \mid \partial_{K_{y}} \Psi\right\rangle-\right.\text { H.c.], }
$$

which, using the formalism above converts to

$$
\sigma_{x y}^{H}=\frac{e^{2}}{h} \lim _{\Delta K_{x} \Delta K_{y} \rightarrow 0} \frac{1}{\Delta K_{x} \Delta K_{y}}\left[\operatorname{Im} \ln \frac{\left\langle\Psi\left|U_{x}\left(\Delta K_{x}\right) U_{y}\left(\Delta K_{y}\right)\right| \Psi\right\rangle}{\left\langle\Psi\left|U_{y}\left(\Delta K_{y}\right)\right| \Psi\right\rangle}+\operatorname{Im} \ln \frac{\left\langle\Psi\left|U_{y}\left(\Delta K_{y}\right) U_{x}\left(-\Delta K_{x}\right)\right| \Psi\right\rangle}{\left\langle\Psi\left|U_{y}\left(\Delta K_{y}\right)\right| \Psi\right\rangle}\right],
$$


where $U_{x}\left(\Delta K_{x}\right)$ and $U_{y}\left(\Delta K_{y}\right)$ are momentum shift operators in the $x$ and $y$ directions. Using the forms of the total momentum shift operators in Eqs. (1) (applicable when the wavefunctions can be written in the coordinate or momentum representations) we can show that in the limit $\Delta K_{x}, \Delta K_{y} \rightarrow 0$ the Hall conductivity takes the form

$$
\sigma_{x y}^{H}=\frac{i e^{2}}{h} \sum_{i}\left\langle\Psi\left|\left[\hat{x}_{i}, \hat{y}_{i}\right]\right| \Psi\right\rangle .
$$

Using Eq. (16) applied to a Landau state one can also derive a quantization condition for the values of the magnetic field at which $\sigma_{x y}^{H}$ must be zero. A Landau level has the form

$$
\psi(x, y)=e^{i k_{x} x} \phi_{n}\left(y-y_{0}\right),
$$

where $y_{0}=k_{x} \frac{\hbar c}{e B}$. As far as the $x$ direction is concerned this function is neither in the momentum nor in the position representations. However, the momentum shift operators can be constructed, considering that a momentum shift in the $x$-direction is also a position shift in the $y$ direction. It is easy to check that in this case

$$
U_{x}\left(\Delta K_{x}\right)=e^{i \Delta K_{x} x} e^{i \Delta Y k_{y}},
$$

with $\Delta Y=\Delta K_{x} \frac{\hbar c}{e B}$. The momentum shift in the $y$ direction remains

$$
U_{y}\left(\Delta K_{y}\right)=e^{i \Delta K_{y} y} .
$$

Applying the shift operators to the Landau state results in

$$
\begin{aligned}
U_{x}\left(\Delta K_{x}\right) U_{y}\left(\Delta K_{y}\right) \psi(x, y) & =e^{i \Delta K_{y}\left(y-y_{0}\right)} e^{i \Delta K_{x} x} \psi(x, y+\Delta y), \\
U_{y}\left(\Delta K_{y}\right) U_{x}\left(-\Delta K_{x}\right) \psi(x, y) & =e^{i \Delta K_{y} \Delta y} e^{i \Delta K_{y}\left(y-y_{0}\right)} e^{i \Delta K_{x} x} \psi(x, y-\Delta y),
\end{aligned}
$$

where $\Delta y=\Delta K_{x} \frac{\hbar c}{e B}$. If $\Delta K_{x} \Delta y=\Delta K_{x} \Delta K_{y} \frac{\hbar c}{e B}=2 \pi M$, with $M$ integer, then the phase in the second of Eqs. (21) is one, and in this case taking the limits $\Delta K_{x}, \Delta K_{y} \rightarrow 0$ results in a Hall conductance of zero. We can take the momentum shifts to be $\Delta K_{x}=q_{x} \frac{2 \pi}{L_{x}}$ and $\Delta K_{y}=q_{y} \frac{2 \pi}{L_{x}}$, with $q_{x}, q_{y}$ integers, which corresponds to equivalent states for the adiabatic case ${ }^{27,28}$ it follows that for a system with $N$ particles the quantization condition is

$$
\frac{e \Phi_{B}}{N h c}=\frac{Q}{M}
$$

where $\Phi_{B}$ denotes the magnetic flux, and $Q$ is an integer. Indeed, the maxima in the Hall resistivity occur ${ }^{15}$ precisely at values of the magnetic flux given by Eq. (22).

\section{CONCLUSION}

In this work it was shown that the zero-frequency conductivity can be expressed in terms of a Berry phase. Subsequently, the conductivity was also expressed in terms of shift operators (total momentum and total position) leading to expressions which provide clear physical insight, as well as a good starting point for numerical work. It was argued that a metallic state is one which is the eigenstate of the total current operator. Such states were also shown to be delocalized. These conclusions were supported by analytic and numerical calculations on a number of examples, both metallic and insulating. If the wavefunction is a linear combination of a total current eigenstate and an insulating wavefunction then a finite dc condutivity results which is smaller than the allowed maximum. Hence, based on the value of the dc conductivity it is possible to distinguish metallic and insulating states from ones in which conducting and insulating states coexist. Subsequently, the formalism was used to express the Hall conductance, and to derive the quantization condition at which the Hall conductance is zero. The condition coincides with the well-known experimental results.

\section{ACKNOWLEDGMENTS}

The author acknowledges a grant from the Turkish agency for basic research (TÜBITAK, Grant No. 112T176).

\section{APPENDIX A: PERTURBED HAMILTONIAN}

The dc conductivity ${ }^{1}$ is proportional to the second derivative of the ground state energy with respect to the Peierls phase $\Phi$ at $\Phi=0$. For a continuous system, taking the mass of charge carriers to be unity, the Hamiltonian has the form

$$
\hat{H}(\Phi)=\sum_{j} \frac{\left(\hat{k}_{j}+\Phi\right)^{2}}{2}+\hat{V} .
$$

In the case of discrete models, one can write

$$
\hat{H}(\Phi)=\hat{T}+\hat{V},
$$

with

$$
\hat{T}(\Phi)=-\sum_{j} t e^{i \Phi} c_{j+1}^{\dagger} c_{j}+\text { H.c. }
$$

(For a detailed discussion, see Refs. 1 and 29.) For both continuous and lattice Hamiltonians, it holds that

$$
H^{\prime}(0)=i[\hat{H}, \hat{X}]=\hat{K}
$$

and

$$
H^{\prime \prime}(0)=i[\hat{K}, \hat{X}],
$$

where $\hat{X}(\hat{K})$ are defined as

$$
\hat{X}=\sum_{j} \hat{x}_{j}, \quad \hat{K}=\sum_{j} \hat{k}_{j},
$$

for continuous systems, and

$$
\hat{X}=\sum_{j} j \hat{n}_{j}, \quad \hat{K}=-i t \sum_{j} c_{j+1}^{\dagger} c_{j}+\text { H.c. },
$$


for lattice models. One can also write $H^{\prime \prime}(0)$ as a sum of one-body operators as

$$
H^{\prime \prime}(0)=-\sum_{j}\left[\hat{k}_{j}, \hat{\partial}_{k_{j}}\right]=-\sum_{j}\left[\hat{\partial}_{x_{j}}, \hat{x}_{j}\right] .
$$

One can also show that

$$
H^{\prime \prime}(0)= \begin{cases}N & \text { for continuous models, } \\ -\hat{T}(0) & \text { for lattice systems. }\end{cases}
$$

One can expand the Hamiltonian and the ground state wave function up to second order as

$$
\begin{gathered}
H(\Phi) \approx H(0)+\Phi H^{\prime}(0)+\frac{\Phi^{2}}{2} H^{\prime \prime}(0), \\
|\Psi(\Phi)\rangle \approx|\Psi(0)\rangle+\Phi\left|\Psi^{\prime}(0)\right\rangle+\frac{\Phi^{2}}{2}\left|\Psi^{\prime \prime}(0)\right\rangle,
\end{gathered}
$$

and express the second derivative of the ground state energy with respect to $\Phi$ at $\Phi=0$ as

$$
\begin{aligned}
\left.\partial_{\Phi}^{2} E(\Phi)\right|_{\Phi=0}= & \left\langle\Psi(0)\left|H^{\prime \prime}(0)\right| \Psi(0)\right\rangle+2\left\langle\Psi^{\prime}(0)\left|H^{\prime}(0)\right| \Psi(0)\right\rangle \\
& +2\left\langle\Psi(0)\left|H^{\prime}(0)\right| \Psi^{\prime}(0)\right\rangle .
\end{aligned}
$$

\section{APPENDIX B: DC CONDUCTIVITY AS A GEOMETRIC PHASE}

In this appendix the dc conductivity is derived in terms of a geometric phase. As shown in Ref. 12 the first derivative of the ground state energy with respect to $\Phi$ for a continuous Hamiltonian is given by

$$
\partial_{\Phi} E(\Phi)=\alpha \Phi-\frac{i}{L} \int_{0}^{L}\left\langle\Psi(X ; \Phi)\left|\partial_{X}\right| \Psi(X ; \Phi)\right\rangle,
$$

where

$$
\alpha= \begin{cases}N & \text { for continuous models, } \\ -\langle\Psi|\hat{T}(0)| \Psi\rangle & \text { for lattice systems. }\end{cases}
$$

Taking the derivative with respect to $\Phi$ and setting $\Phi$ to zero results in

$$
\begin{aligned}
\left.\partial_{\Phi}^{2} E(\Phi)\right|_{\Phi=0}= & \alpha-\frac{i}{L} \int_{0}^{L} d X\left[\left\langle\partial_{\Phi} \Psi(X)\left|\partial_{X}\right| \Psi(X)\right\rangle\right. \\
& \left.+\left\langle\Psi(X)\left|\partial_{X}\right| \partial_{\Phi} \Psi(X)\right\rangle\right] .
\end{aligned}
$$

Since $\Phi$ corresponds to a shift in the crystal momentum $K$ the derivative with respect to $\Phi$ can be replaced with a derivative with respect to $K$. Subsequently, an average over $K$ can be taken, resulting in

$$
\left.\partial_{\Phi}^{2} E(\Phi)\right|_{\Phi=0}=\alpha+\gamma
$$

with

$$
\gamma=-\frac{i}{m 2 \pi} \int_{0}^{L} \int_{-\pi / L}^{\pi / L} d X d K\left[\left\langle\partial_{K} \Psi \mid \partial_{X} \Psi\right\rangle-\left\langle\partial_{X} \Psi \mid \partial_{K} \Psi\right\rangle\right] .
$$

The quantity $\gamma$ in Eq. (B4) is a surface integral over a Berry curvature, which can be converted into a line integral around the included surface via the Stokes theorem, as for the Hall conductivity. ${ }^{14}$
The quantity $\alpha$ can be written with the help of Eq. (A8) as

$$
\alpha=i \sum_{j}\left\langle\Psi\left|\left[\partial_{x_{j}}, \partial_{k_{j}}\right]\right| \Psi\right\rangle .
$$

In other words, the conductivity corresponds to the difference between the sum of one body commutators of the position and momenta and the commutator of the total position and total momentum.

\section{APPENDIX C: DC CONDUCTIVITY IN TERMS OF SHIFT OPERATORS}

Our starting point is the current ${ }^{12}$ written in terms of shift operators, ${ }^{16}$

$$
\partial_{\Phi} E(\Phi)=\alpha \Phi-\frac{1}{\Delta X} \operatorname{Im} \ln \left\langle\Psi(\Phi)\left|e^{i \Delta X \hat{K}}\right| \Psi(\Phi)\right\rangle .
$$

Taking the derivative with respect to $\Phi$ results in

$$
\partial_{\Phi} E(\Phi)=\alpha+\gamma
$$

with

$$
\begin{aligned}
\gamma= & \frac{1}{\Delta X} \operatorname{Im}\left[\frac{\left\langle\partial_{\Phi} \Psi(\Phi)\left|e^{i \Delta X \hat{K}}\right| \Psi(\Phi)\right\rangle}{\left\langle\Psi(\Phi)\left|e^{i \Delta X \hat{K}}\right| \Psi(\Phi)\right\rangle}\right. \\
& \left.+\frac{\left\langle\Psi(\Phi)\left|e^{i \Delta X \hat{K}}\right| \partial_{\Phi} \Psi(\Phi)\right\rangle}{\left\langle\Psi(\Phi)\left|e^{i \Delta X \hat{K}}\right| \Psi(\Phi)\right\rangle}\right] .
\end{aligned}
$$

We can set the derivative in $\Phi$ equal to the derivative in the crystal momentum, and set $\Phi=0$. For now we will consider only the first term in Eq. (C3), but the steps for the second term are essentially identical. We can write this term as

$$
\frac{1}{\Delta X \Delta K} \operatorname{Im}\left[\frac{\Delta K\left\langle\partial_{K} \Psi(0)\left|e^{i \Delta X \hat{K}}\right| \Psi(0)\right\rangle}{\left\langle\Psi(0)\left|e^{i \Delta X \hat{K}}\right| \Psi(0)\right\rangle}\right],
$$

where we have divided and multiplied by $\Delta K$. For small $\Delta K$ we can replace this term with

$$
\frac{1}{\Delta X \Delta K} \operatorname{Im} \ln \left[1+\frac{\Delta K\left\langle\partial_{K} \Psi(0)\left|e^{i \Delta X \hat{K}}\right| \Psi(0)\right\rangle}{\left\langle\Psi(0)\left|e^{i \Delta X \hat{K}}\right| \Psi(0)\right\rangle}\right],
$$

which can be converted to

$$
\frac{1}{\Delta X \Delta K} \operatorname{Im} \ln \left[\frac{\left\langle\Psi(\Delta K)\left|e^{i \Delta X \hat{K}}\right| \Psi(0)\right\rangle}{\left\langle\Psi(0)\left|e^{i \Delta X \hat{K}}\right| \Psi(0)\right\rangle}\right],
$$

and using the total momentum shift operator results in

$$
\frac{1}{\Delta X \Delta K} \operatorname{Im} \ln \left[\frac{\left\langle\Psi\left|e^{i \Delta K \hat{X}} e^{i \Delta X \hat{K}}\right| \Psi\right\rangle}{\left\langle\Psi\left|e^{i \Delta X \hat{K}}\right| \Psi\right\rangle}\right] .
$$

Applying exactly the same steps to the second term of Eq. (C3) results in

$$
\begin{aligned}
\gamma= & \frac{1}{\Delta X \Delta K}\left[\operatorname{Im} \ln \left(\frac{\left\langle\Psi\left|e^{i \Delta K \hat{X}} e^{i \Delta X \hat{K}}\right| \Psi\right\rangle}{\left\langle\Psi\left|e^{i \Delta X \hat{K}}\right| \Psi\right\rangle}\right)\right. \\
& \left.+\operatorname{Im} \ln \left(\frac{\left\langle\Psi\left|e^{i \Delta X \hat{K}} e^{-i \Delta K \hat{X}}\right| \Psi\right\rangle}{\left\langle\Psi\left|e^{i \Delta X \hat{K}}\right| \Psi\right\rangle}\right)\right],
\end{aligned}
$$

which is the discretized form for the Drude weight. 
${ }^{1}$ W. Kohn, Phys. Rev. 133, A171 (1964).

${ }^{2}$ E. S. Fois, A. Selloni, M. Parrinello, and R. Car, J. Phys. Chem. 92, 3268 (1988).

${ }^{3}$ A. Selloni, P. Carnevali, R. Car, and M. Parrinello, Phys. Rev. Lett. 59, 823 (1987).

${ }^{4}$ R. Resta, Rev. Mod. Phys. 66, 899 (1994).

${ }^{5}$ R. D. King-Smith and D. Vanderbilt, Phys. Rev. B 47, 1651 (1993).

${ }^{6}$ S. Pancharatnam, Proc. Indian Acad. Sci. A 44, 247 (1956).

${ }^{7}$ M. V. Berry, Proc. R. Soc. A 392, 45 (1984).

${ }^{8}$ D. Xiao, M.-C. Chang, and Q. Niu, Rev. Mod. Phys. 82, 1959 (2010).

${ }^{9}$ R. Resta, Phys. Rev. Lett. 80, 1800 (1998).

${ }^{10}$ R. Resta and S. Sorella, Phys. Rev. Lett. 82, 370 (1999).

${ }^{11}$ K. Moulopoulos and N. W. Ashcroft, Phys. Rev. B 45, 11518 (1992).

${ }^{12}$ B. Hetényi, J. Phys. Soc. Jpn. 81, 124711 (2012).

${ }^{13}$ J.-H. Zhou, H. Jiang, Q. Niu, and J.-R. Shi, Chin. Phys. Lett. 30, 027101 (2013).

${ }^{14}$ D. J. Thouless, M. Kohmoto, M. P. Nightingale, and M. den Nijs, Phys. Rev. Lett. 49, 405 (1982).
${ }^{15}$ H. L. Störmer, D. C. Tsui, and A. C. Gossard, Rev. Mod Phys. 71, S298 (1999).

${ }^{16}$ B. Hetényi, J. Phys. A 42, 412003 (2009).

${ }^{17}$ B. Hetényi, J. Phys. Soc. Jpn. 81, 023701 (2012).

${ }^{18}$ M. C. Gutzwiller, Phys. Rev. Lett. 10, 159 (1963).

${ }^{19}$ J. Hubbard, Proc. R. Soc. A 276, 238 (1963).

${ }^{20}$ J. Kanamori, Prog. Theor. Phys. 30, 275 (1963).

${ }^{21}$ A. J. Millis and S. N. Coppersmith, Phys. Rev. B 43, 13770 (1991).

${ }^{22}$ M. Dzierzawa, D. Baeriswyl, and L. M. Martelo, Helv. Phys. Acta 70, 124 (1997).

${ }^{23}$ B. Valenzuela, S. Fratini, and D. Baeriswyl, Phys. Rev. B 68, 045112 (2003).

${ }^{24}$ P. W. Anderson, Phys. Rev. 109, 1492 (1958).

${ }^{25}$ T. Neupert, L. Santos, C. Chamon, and C. Mudry, Phys. Rev. B 86, 165133 (2012).

${ }^{26}$ T. Neupert, L. Santos, S. Ryu, C. Chamon, and C. Mudry, Phys. Rev. B 86, 035125 (2012).

${ }^{27}$ R. B. Laughlin, Phys. Rev. B 23, 5632 (1981).

${ }^{28}$ N. Byers and C. N. Yang, Phys. Rev. Lett. 7, 46 (1961).

${ }^{29}$ F. H. L. Essler, H. Frahm, F. Göhmann, A. Klümper, and V. E. Korepin, The One-Dimensional Hubbard Model (Cambridge University Press, Cambridge, UK, 2005). 